\title{
Bubble Identification Based on High Speed Videometry Data: Algorithm and Validation
}

\author{
Carlos E.F. do Amaral, Rafael F. Alves, Marco J. da Silva, Lúcia V.R. Arruda, \\ Leyza B. Dorini, and Rigoberto E.M. Morales
}

Federal University of Technology - Paraná. 3165 Sete de Setembro Av., CEP: 80230-901

Curitiba-Paraná, Brazil

\begin{abstract}
The simultaneous flow of gas and liquid in a pipe is commonly found in several industrial activities, such as crude oil extraction and processing. In order to analyze this (two-phase) flow, many measurement techniques have been proposed, including X-ray, ultrasound, impedance and optical measurement. In this context, although the high speed videometry use is limited in practical cases, it is an important tool to validate other methods in experimental essays. Approaches based on image segmentation have already been considered to analyze gas-liquid flows along vertical pipes, but just a few have focused horizontal experiments, which are also widely found in many applications. This article describes a new technique developed to automatically measure the bubble volume through video analysis. The tests carried out considering horizontal air-water flow images yielded results with good correlation with known volume data, thus showing that the measurements are accurate enough to be considered for the validation of other technologies.
\end{abstract}

Keywords: Two-phase flow, watershed segmentation, bubble identification.

\section{Introduction}

Gas-liquid two-phase flows are present in a wide range of industrial cases, including food, nuclear, aerospace, geothermal and petroleum industry. In these cases, such flows are typically constrained to pipes or vessels and may have an important role in determining the safety and efficiency of the plant processes [1]. Thus, detailed information about flow behavior under controlled conditions with accurate non- or minimal intrusive measurement techniques is essential for flow modeling and prediction.

The measurement and imaging of two-phase flows have received much attention recently. Several techniques have already been used and developed to analyze the phenomena in two-phase flow, including high-speed videometry [2], capacitive probes [3] and ultrasound [4], X-ray tomographic imaging [5] and impedanciometry [6]. High-speed videometry offers many interesting characteristics for laboratories essays, such as non-invasiveness, advances in instrumentation technology and signal processing algorithms [7]. Image processing techniques also constitute a powerful tool to study the two-phase flow phenomena, being typically non-intrusive and relatively simple to design and implement. To separate the different substances (phases) flowing inside the pipe, filtering [2] and wavelets [8] have been applied. Although 
many works have addressed vertical applications, just a few studies have been done in horizontal cases [9].

In this paper, we propose an algorithm to automatically extract quantitative information of air bubble volumes from high-speed camera acquisitions in horizontal twophase flows. The obtained images are processed using mathematical morphology operations and the watershed transform. The obtained results have shown to be accurate when compared to known volume data and, thus, can be considered for the validation of other technologies.

\section{Experimental Setup}

The experimental loop (Fig. 1), located in the Thermal Sciences Laboratory (LACIT) at the Federal University of Technology - Paraná (UTFPR), is able to produce different flow patterns of air and tap water mixtures. In this paper, we consider only the slug pattern, which has as main characteristic the alternation between bubbles and a liquid region between them. This intermittent regime has large bubbles, called Taylor bubbles [7].

The horizontal acrylic pipe has an internal diameter of 26 millimeters and is 9 meters long. The water flow rate is independently measured through a Coriolis flow meter for water. The air is provided by a system that controls the exactly gas volume before the mixing entrance. In the tube exit, a separator/reservatory expels the air to the atmosphere and store the water.

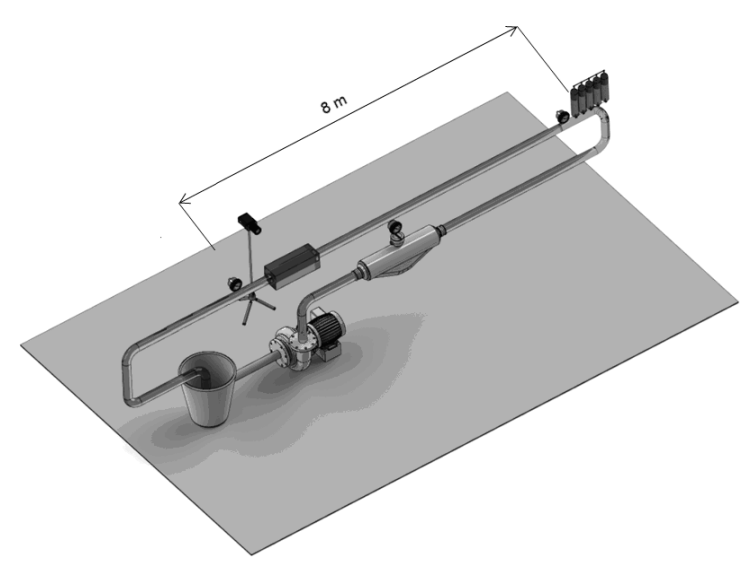

Fig. 1. Schematic representation from the experimental plant

The control of the temperature of the phase, as well as of the pressure of the twophase mixture at the entrance and in the measurement point is made by a host computer connected to sensors via Foundation Field Bus. In such a way, it is possible to measure the total air volume at the video images based on the difference between the pressures measured at the pipe entrance and at the measurement position, as defined by Eq. (1):

$$
P_{1} V_{1}=P_{2} V_{2}
$$


where $P_{1}$ and $P_{2}$ denote the pressure in the entrance and in the measurement position and $V_{l}$ and $V_{2}$ represent, respectively, the volume (in $\mathrm{m}^{3}$ ) of the gas-system input (which may vary for each measurement) and of the position where the sensors are placed.

The two-phase flow images were acquired using a high speed camera (NanoSense MKIII, Dantec Dynamics A/S) at a resolution of 320x500 pixels and with a frame rate of $60 \mathrm{~Hz}$. A $140 \mu$ s exposure time was chosen to avoid blurry images. A rectangular transparent acrylic box (200 $\mathrm{mm} \times 100 \mathrm{~mm} \times 100 \mathrm{~mm})$ filled with water was used to better match the refraction indices of the water and the acrylic pipe, thus reducing light refraction in the pipe borders. A strong and pulsed illumination source was placed $100 \mathrm{~mm}$ behind this acrylic box. The light source contains 19 high light bright leds (MotionLEDs - IDT). To ensure an uniform illumination all over the test section, a diffuser was placed between the light source and the acrylic box.

A total of 10 injection volumes, repeated 10 times, have been measured by the high-speed camera. For each experimental condition, an air volume were injected in the pipe at a constant liquid flow rate set up to $1911.34 \mathrm{l} / \mathrm{h}$. The measurements were stored over 10 seconds, time enough to acquire the gas passing the test section. The proposed methodology was implemented using the MatLab plataform, due the great number of functions already available for statistics and image treatment. The running time to process all data $\left(9,6 \times 10^{9}\right.$ pixels) was 2 hours and 42 minutes on an Intel Quad Core 2 i7 (@2.66 GHz) with 6 Gb of RAM memory and running Windows 764 bits.

\section{Image Segmentation and Bubble Detection}

In a morphological framework, considered in the approach proposed in this paper, gray-scale image segmentation is typically implemented by first extracting markers of the significant structures, and then using the watershed transform to extract the contours of these structures as accurately as possible.

Intuitively, the watershed transform can be defined as a flood simulation, where an image is seen as a topographical surface whose pixel values correspond to the elevation at that point, as illustrated in Fig. 2(a). The flooding process starts with the water filling markers such as the image minima (or "valleys"). When the flooding of two minima met, a dam is built to identify this boundary, allowing segmentation of objects in different regions [11]. Fig. 2 (b) shows the top view of the image after watershed treatment.

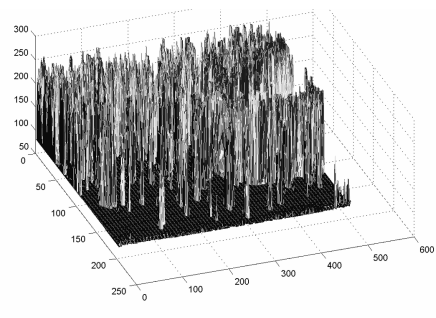

(a)

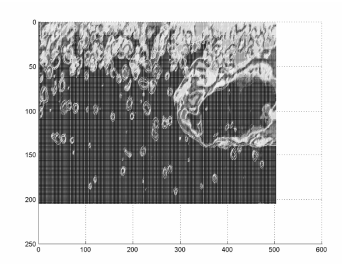

(b)

Fig. 2. (a) $3 D$ view of the watershed performance after the H-minima transform (b) Top view of the same landscape after flooding process 
However, image extrema (frequently used as markers in the watershed transform) can correspond to insignificant structures or noise, conducing to the over-segmentation problem. To prevent it, image extrema are usually selected according to some criteria, such as contrast, area and so forth. A typical approach consists on considering the hmaxima (h-minima) transform to suppress all image maxima (minima), whose contrast is lower than a specified value $h$, and use the extended extrema as markers [10].

Fig. 3 depicts an example. The watershed transform is applied to the original high speed camera image (Fig 3(a)) considering a set of extended extrema as markers, yielding the segmentation shown in Fig. 3(b). Since the test images correspond to the slug flow pattern, we can assume that the main air volume is found in the Taylor bubble body. Thus, we mark as interest regions only that with a segmented area larger than $4 \%$ of the total frame surface, as illustrated in Fig. 3 (c).

Observe that the algorithm may also mark regions that correspond to the gap between dispersed bubbles, which does not contribute significantly to the total air volume. To avoid this problem, we discard all regions that are not in contact with the right or left boards of the image. Also note that small parts of the main bubble may be segmented separately. In order to still consider these areas, the software assigns the regions connected to the main bubble as part of the resulting segmentation, as presented in Fig. 3 (d).

(a)

(b)

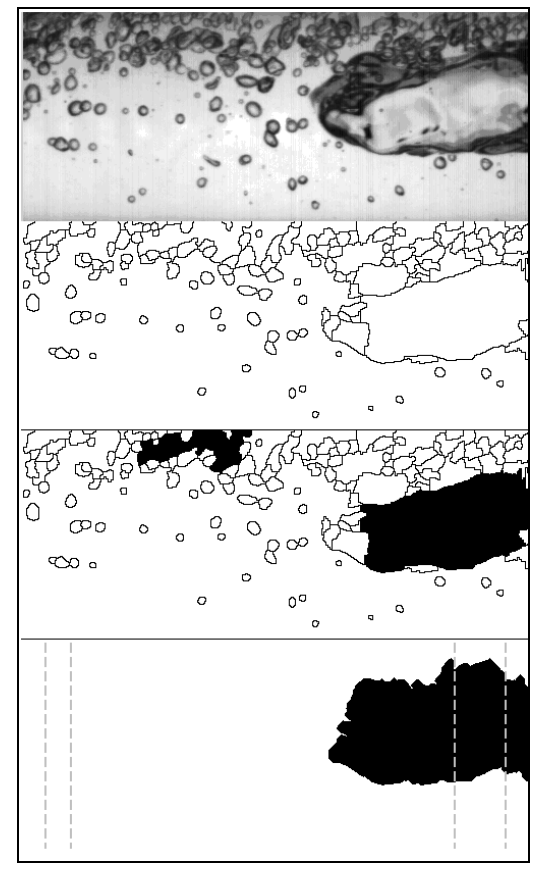

Fig. 3. Detection of bubbles borders based on the watershed transform segmentation results

Based on this procedure, it is possible to determine the bubble edges and, further, the bubble nose coordinates, thus allowing the volume computation, as described in the following. 


\section{Bubble Volume Calculation}

To approximate the bubble volume, it is necessary to build a panoramic image. One example is illustrated in Fig. 4.

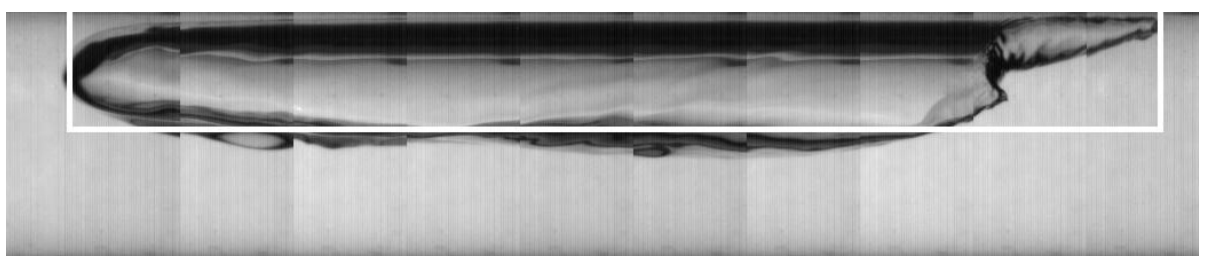

Fig. 4. Gas bubble reconstructed from 8 frames acquisition and marked with bubble nose, tail and mean high coordinates

To perform this task, the images used in the test section are subjected to a reconstruction process, which takes into account the velocity of the bubble to join the frames and produce the panoramic image. The velocity of image data can be computed based on the difference between the nose frames cordinates, as defined in Eq. (2).

$$
U_{B}=\frac{\Delta K_{P}}{\Delta_{P}} \cdot\left(P_{S}\right) \cdot\left(F_{R}\right)
$$

where $U_{B}$ is the bubble nose velocity (meters per second), $\Delta K_{P}$ is the difference in pixels between the $K$ position on the considered frames (pixels), $\Delta_{P}$ is the number of frames showing the bubble nose (frames), $P_{S}$ is the pixel size (meters per pixel) calculated by dividing the field of view length by the number of horizontal pixels in the frame and $F_{R}$ is the frame rate (frames/second).

Using the image segmentation approach detailed in Section 3, it is possible to estimate the bubble height, denoted here by h. This parameter is illustrated as the highlighted segment in Fig. 5.

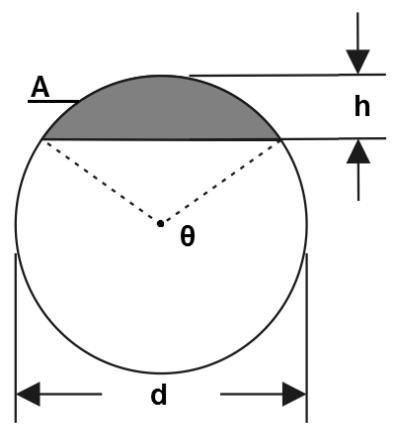

Fig. 5. Determination of bubble frame area where $d$ is the pipe diameter, $\mathrm{h}$ is the air bubble high 
The angle $\theta$ (rad) from the arc can be found using the Eq. (3):

$$
\theta=2 \arcsin \left(1-\frac{2 h}{d}\right)
$$

where $d$ (meters) is the diameter from the pipe and $h$ (meters) the height of gas column.

After computing the circular segment shape, it is assumed that the bubble has the same format from the beginning to the end. Then, the approximate bubble volume is calculated as shown in Eq. (4).

$$
V=\left(\left(1-\frac{(\theta-\sin (\theta))}{2 \pi}\right) \cdot \frac{\pi d^{2}}{4}\right) \cdot n
$$

where $d$ (meters) is the diameter from the pipe, $n$ is the number of the pixels in the $\mathrm{k}$ axis that compose the bubble and $V\left(\mathrm{~m}^{3}\right)$ the bubble volume.

We emphasize that this method is an approximation for the regular shape of bubbles and only works for horizontal flows, where the gravity forces the gas bubble at the top of pipe.

\section{$5 \quad$ Results and Discussion}

Fig. 6 shows the bubble volume results obtained using the proposed approach compared with the correct reference volume from gas injection system considering a $\pm 10 \%$ error boundaries. One can notice a video treatment tendency to decrease the real bubble volume in around $10 \%$. This can be explained due the fact that small dispersed bubbles may be not detected by the computer algorithm.

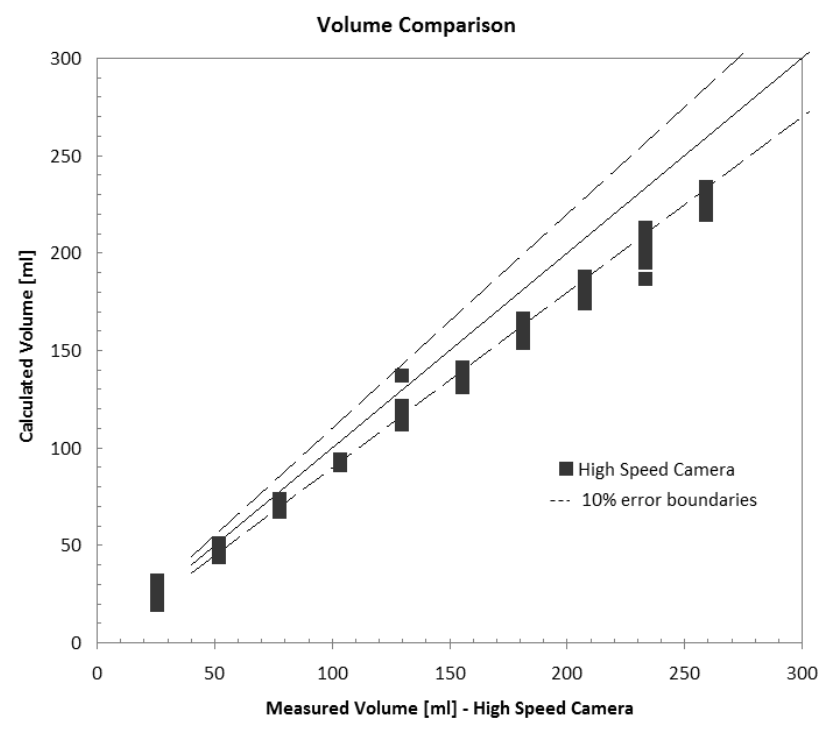

Fig. 6. Bubbles volume comparison between High Speed Camera and know amount of air mixed to the water 


\section{Conclusion}

The operation principle of image treatment was reviewed, as well as the steps for extraction of air flow volume based in the high speed videometry data. We have developed and tested an algorithm for bubble segmentation in gas-liquid flows based in the watershed transform and on mathematical morphology operations. Subsequently, derived parameters such as bubble size and velocity distributions can be obtained. The develop technique obtained satisfactory results for bubbles volume measurements when compared with the real values, validating the proposed algorithm.

\section{References}

1. Shoham, O.: Mechanistic Modeling of Gas-Liquid Two-phase Flow in Pipes. Society of Petroleum (2006)

2. Mayor, T., Pinto, a., Campos, J.: Vertical slug flow in laminar regime in the liquid and turbulent regime in the bubble wake-Comparison with fully turbulent and fully laminar regimes. Chemical Engineering Science 63, 3614-3631 (2008)

3. Ahmed, W., Ismail, B.: Innovative Techniques For Two-Phase Flow Measurements. Recent Patents on Electrical Engineering 1, 1-13 (2008)

4. Skwarek, V., Windorfer, H., Hans, V.: Measuring pulsating flow with ultrasound. Measurement 29, 225-236 (2001)

5. Hervieu, E., Jouet, E., Desbat, L.: Development and validation of an X-ray tomograph for two-phase flow. Annals of the New York Academy of Sciences 972, 87-94 (2002)

6. Wang, M.: Impedance mapping of particulate multiphase flows. Flow Measurement and Instrumentation 16, 183-189

7. Falcone, G., Hewitt, G.F., Alimonti, C.: Multiphase Flow Metering: Principles and Applications. Elsevier Science \& Technology (2009)

8. Guo, F., Yang, Y., Chen, B., Guo, L.: A novel multi-scale edge detection technique based on wavelet analysis with application in multiphase flows. Powder Technology 202, 171177 (2010)

9. Hout, R.V., Barnea, D., Shemer, L.: Translational velocities of elongated bubbles in continuous slug flow 28, 1333-1350 (2002)

10. Soille, P.: Morphological Image Analysis: Principles and Applications. Springer (2003)

11. Pratikakis, I.: Low level image partitioning guided by the gradient watershed hierarchy. Signal Processing 75, 173-195 (1999) 